\title{
Insertion of A Baire-one Function
}

\author{
MAJID MIRMIRAN \\ Department of Mathematics, University of Isfahan, Isfahan , 81746-73441, Iran \\ *Corresponding Author: mirmir@sci.ui.ac.ir \\ Copyright (C)2013 Horizon Research Publishing All rights reserved.
}

\begin{abstract}
A necessary and sufficient condition in terms of lower cut sets are given for the insertion of a Baire-one function between two comparable real-valued functions on the topological spaces that $\Lambda$-sets are $G_{\delta}$-sets.
\end{abstract}

Keywords Insertion, Strong binary relation, Baireone function, $\Lambda$-sets, Lower cut set.

\section{Introduction}

A generalized class of closed sets was considered by Maki in 1986 [5]. He investigated the sets that can be represented as union of closed sets and called them $V$-sets. Complements of $V$-sets, i.e., sets that are intersection of open sets are called $\Lambda$-sets [5].

Results of Katětov [2] , [3] concerning binary relations and the concept of an indefinite lower cut set for a realvalued function, which is due to Brooks [1], are used in order to give a necessary and sufficient condition for the insertion of a Baire-one function between two comparable real-valued functions on the topological spaces that $\Lambda$-sets are $G_{\delta}$-sets.

A real-valued function $f$ defined on a topological space $X$ is called Baire-one if the preimage of every open subset of $R$ is a $F_{\sigma}$-set in $X$.

If $g$ and $f$ are real-valued functions defined on a space $X$, we write $g \leq f$ (resp. $g<f$ ) in case $g(x) \leq f(x)$ (resp. $g(x)<f(x)$ ) for all $x$ in $X$.

The following definitions are modifications of conditions considered in [4].

A property $P$ defined relative to a real-valued function on a topological space is a $B_{1}$-property provided that any constant function has property $P$ and provided that the sum of a function with property $P$ and any Baire-one function also has property $P$. If $P_{1}$ and $P_{2}$ are $B_{1}$-properties, the following terminology is used: (i) A space $X$ has the weak $B_{1}$-insertion property for $\left(P_{1}, P_{2}\right)$ iff for any functions $g$ and $f$ on $X$ such that $g \leq f, g$ has property $P_{1}$ and $f$ has property $P_{2}$, then there exists a Baire-one function $h$ such that $g \leq h \leq f$. (ii) A space $X$ has the $B_{1}$-insertion property for $\left(P_{1}, P_{2}\right)$ iff for any functions $g$ and $f$ on $X$ such that $g<f, g$ has property $P_{1}$ and $f$ has property $P_{2}$, then there exists a Baire-one function $h$ such that $g<h<f$.
In this paper, for a topological space that $\Lambda$-sets are $G_{\delta}$-sets, is given a sufficient condition for the weak $B_{1}$-insertion property. Also for a space with the weak $B_{1}$-insertion property, we give a necessary and sufficient condition for the space to have the $B_{1}$-insertion property. Several insertion theorems are obtained as corollaries of these results.

\section{The main results}

Before giving a sufficient condition for insertability of a Baire-one function, the necessary definitions and terminology are stated.

Definition. Let $A$ be a subset of a topological space $(X, \tau)$. We define the subsets $A^{\Lambda}$ and $A^{V}$ as follows: $A^{\Lambda}=\cap\{O: O \supseteq A, O \in(X, \tau)\}$ and $A^{V}=\cup\{F: F \subseteq$ $\left.A, F^{c} \in(X, \tau)\right\}$.

$A^{\Lambda}$ is called kernel of $A$.

The following first two definitions are modifications of conditions considered in [2], [3].

Definition. If $\rho$ is a binary relation in a set $S$ then $\bar{\rho}$ is defined as follows: $x \bar{\rho} y$ if and only if $y \rho \nu$ implies $x \rho \nu$ and $u \rho x$ implies $u \rho y$ for any $u$ and $v$ in $S$.

Definition. A binary relation $\rho$ in the power set $P(X)$ of a topological space $X$ is called a strong binary relation in $P(X)$ in case $\rho$ satisfies each of the following conditions:

1) If $A_{i} \rho B_{j}$ for any $i \in\{1, \ldots, m\}$ and for any $j \in$ $\{1, \ldots, n\}$, then there exists a set $C$ in $P(X)$ such that $A_{i} \rho C$ and $C \rho B_{j}$ for any $i \in\{1, \ldots, m\}$ and any $j \in\{1, \ldots, n\}$.

2) If $A \subseteq B$, then $A \bar{\rho} B$.

3) If $A \rho B$, then $A^{\Lambda} \subseteq B$ and $A \subseteq B^{V}$.

The concept of a lower indefinite cut set for a real-valued function was defined by Brooks [1] as follows:

Definition. If $f$ is a real-valued function defined on a space $X$ and if $\{x \in X: f(x)<\ell\} \subseteq A(f, \ell) \subseteq\{x \in X$ : $f(x) \leq \ell\}$ for a real number $\ell$, then $A(f, \ell)$ is a lower indefinite cut set in the domain of $f$ at the level $\ell$.

We now give the following main results:

Theorem 2.1. Let $g$ and $f$ be real-valued functions on the topological space $X$, that $\Lambda$-sets in $X$ are $G_{\delta}$-sets, 
with $g \leq f$. If there exists a strong binary relation $\rho$ on the power set of $X$ and if there exist lower indefinite cut sets $A(f, t)$ and $A(g, t)$ in the domain of $f$ and $g$ at the level $t$ for each rational number $t$ such that if $t_{1}<t_{2}$ then $A\left(f, t_{1}\right) \rho A\left(g, t_{2}\right)$, then there exists a Baire-one function $h$ defined on $X$ such that $g \leq h \leq f$.

Proof. Let $g$ and $f$ be real-valued functions defined on the $X$ such that $g \leq f$. By hypothesis there exists a strong binary relation $\rho$ on the power set of $X$ and there exist lower indefinite cut sets $A(f, t)$ and $A(g, t)$ in the domain of $f$ and $g$ at the level $t$ for each rational number $t$ such that if $t_{1}<t_{2}$ then $A\left(f, t_{1}\right) \rho A\left(g, t_{2}\right)$.

Define functions $F$ and $G$ mapping the rational numbers $Q$ into the power set of $X$ by $F(t)=A(f, t)$ and $G(t)=A(g, t)$. If $t_{1}$ and $t_{2}$ are any elements of $Q$ with $t_{1}<t_{2}$, then $F\left(t_{1}\right) \bar{\rho} F\left(t_{2}\right), G\left(t_{1}\right) \bar{\rho} G\left(t_{2}\right)$, and $F\left(t_{1}\right) \rho G\left(t_{2}\right)$. By Lemmas 1 and 2 of [3] it follows that there exists a function $H$ mapping $Q$ into the power set of $X$ such that if $t_{1}$ and $t_{2}$ are any rational numbers with $t_{1}<t_{2}$, then $F\left(t_{1}\right) \rho H\left(t_{2}\right), H\left(t_{1}\right) \rho H\left(t_{2}\right)$ and $H\left(t_{1}\right) \rho G\left(t_{2}\right)$.

For any $x$ in $X$, let $h(x)=\inf \{t \in Q: x \in H(t)\}$.

We first verify that $g \leq h \leq f$ : If $x$ is in $H(t)$ then $x$ is in $G\left(t^{\prime}\right)$ for any $t^{\prime}>t$; since $x$ in $G\left(t^{\prime}\right)=A\left(g, t^{\prime}\right)$ implies that $g(x) \leq t^{\prime}$, it follows that $g(x) \leq t$. Hence $g \leq h$. If $x$ is not in $H(t)$, then $x$ is not in $F\left(t^{\prime}\right)$ for any $t^{\prime}<t$; since $x$ is not in $F\left(t^{\prime}\right)=A\left(f, t^{\prime}\right)$ implies that $f(x)>t^{\prime}$, it follows that $f(x) \geq t$. Hence $h \leq f$.

Also, for any rational numbers $t_{1}$ and $t_{2}$ with $t_{1}<t_{2}$, we have $h^{-1}\left(t_{1}, t_{2}\right)=H\left(t_{2}\right)^{V} \backslash H\left(t_{1}\right)^{\Lambda}$. Hence $h^{-1}\left(t_{1}, t_{2}\right)$ is a $F_{\sigma}$-set in $X$, i.e., $h$ is a Baire-one function on $X$.

The above proof used the technique of theorem 1 of[2].

Theorem 2.2. Let $P_{1}$ and $P_{2}$ be $B_{1}$-property and $X$ be a space that satisfies the weak $B_{1}$-insertion property for $\left(P_{1}, P_{2}\right)$. Also assume that $g$ and $f$ are functions on $X$ such that $g<f, g$ has property $P_{1}$ and $f$ has property $P_{2}$. The space $X$ has the $B_{1}$-insertion property for $\left(P_{1}, P_{2}\right)$ iff there exist lower cut sets $A\left(f-g, 3^{-n+1}\right)$ and there exists a decreasing sequence $\left\{D_{n}\right\}$ of subsets of $X$ with empty intersection and such that for each $n, X \backslash D_{n}$ and $A\left(f-g, 3^{-n+1}\right)$ are completely separated by Baireone functions.

Proof. Assume that $X$ has the weak $B_{1}$-insertion property for $\left(P_{1}, P_{2}\right)$. Let $g$ and $f$ be functions such that $g<f, g$ has property $P_{1}$ and $f$ has property $P_{2}$. By hypothesis there exist lower cut sets $A\left(f-g, 3^{-n+1}\right)$ and there exists a sequence $\left(D_{n}\right)$ such that $\bigcap_{n=1}^{\infty} D_{n}=\emptyset$ and such that for each $n, X \backslash D_{n}$ and $A\left(f-g, 3^{-n+1}\right)$ are completely separated by Baire-one functions. Let $k_{n}$ be a Baire-one function such that $k_{n}=0$ on $A\left(f-g, 3^{-n+1}\right)$ and $k_{n}=1$ on $X \backslash D_{n}$. Let a function $k$ on $X$ be defined by

$$
k(x)=1 / 2 \sum_{n=1}^{\infty} 3^{-n} k_{n}(x) .
$$

By the Cauchy condition and the properties of Baireone, the function $k$ is a Baire-one function. Since $\bigcap_{n=1}^{\infty} D_{n}=\emptyset$ and since $k_{n}=1$ on $X \backslash D_{n}$, it follows that $0<k$. Also $2 k<f-g$ : In order to see this, observe first that if $x$ is in $A\left(f-g, 3^{-n+1}\right)$, then $k(x) \leq 1 / 4\left(3^{-n}\right)$. If $x$ is any point in $X$, then $x \notin A(f-g, 1)$ or for some $n$,

$$
x \in A\left(f-g, 3^{-n+1}\right)-A\left(f-g, 3^{-n}\right) ;
$$

in the former case $2 k(x)<1$, and in the latter $2 k(x) \leq$ $1 / 2\left(3^{-n}\right)<f(x)-g(x)$. Thus if $f_{1}=f-k$ and if $g_{1}=g+k$, then $g<g_{1}<f_{1}<f$. Since $P_{1}$ and $P_{2}$ are $B_{1}$-properties, then $g_{1}$ has property $P_{1}$ and $f_{1}$ has property $P_{2}$. Since $X$ has the weak $B_{1}$-insertion property for $\left(P_{1}, P_{2}\right)$, then there exists a Baire-one function $h$ such that $g_{1} \leq h \leq f_{1}$. Thus $g<h<f$, it follows that $X$ satisfies the $B_{1}$-insertion property for $\left(P_{1}, P_{2}\right)$. (The technique of this proof is by Katětov[3]).

Conversely, let $g$ and $f$ be functions on $X$ such that $g$ has property $P_{1}, f$ has property $P_{2}$ and $g<f$. By hypothesis, there exists a Baire-one function $h$ such that $g<h<f$.We follow an idea contained in Lane [4]. Since the constant function 0 has property $P_{1}$, since $f-h$ has property $P_{2}$, and since $X$ has the $B_{1}$-insertion property for $\left(P_{1}, P_{2}\right)$, then there exists a Baire-one function $k$ such that $0<k<f-h$. Let $A\left(f-g, 3^{-n+1}\right)$ be any lower cut set for $f-g$ and let $D_{n}=\left\{x \in X: k(x)<3^{-n+2}\right\}$. Since $k>0$ it follows that $\bigcap_{n=1}^{\infty} D_{n}=\emptyset$. Since

$$
A\left(f-g, 3^{-n+1}\right) \subseteq\left\{x \in X:(f-g)(x) \leq 3^{-n+1}\right\} \subseteq
$$

$$
\left\{x \in X: k(x) \leq 3^{-n+1}\right\}
$$

and since $\left\{x \in X: k(x) \leq 3^{-n+1}\right\}$ and $\left\{x \in X: k(x) \geq 3^{-n+2}\right\}=X \backslash D_{n}$ are completely separated by $\sup \left\{3^{-n+1}, \inf \left\{k, 3^{-n+2}\right\}\right\}$ that is a Baire-one function, it follows that for each $n, A\left(f-g, 3^{-n+1}\right)$ and $X \backslash D_{n}$ are completely separated by Baire-one functions.

\subsection{Applications}

Definition. A real-valued function $f$ defined on a space $X$ is called upper semi-Baire-one (resp. lower semiBaire-one) if $f^{-1}(-\infty, t)$ (resp. $\left.f^{-1}(t,+\infty)\right)$ is a $F_{\sigma}$-set for any real number $t$.

The abbreviations $u s c, l s c, u s B_{1}$ and $l s B_{1}$ are used for upper semicontinuous, lower semicontinuous, upper semi-Baire-one, and lower semi-Baire-one, respectively.

Remark 1. [2], [3]. A space $X$ has the weak $c$-insertion property for $(u s c, l s c)$ iff $X$ is normal.

Before stating the consequences of theorems 2.1, 2.2, we suppose that $X$ is a topological space that $\Lambda$-sets are $G_{\delta}-$ sets.

Corollary 3.1. For each pair of disjoint $G_{\delta}$-sets $G_{1}, G_{2}$, there are two $F_{\sigma}$-sets $F_{1}$ and $F_{2}$ such that $G_{1} \subseteq F_{1}, G_{2} \subseteq F_{2}$ and $F_{1} \cap F_{2}=\emptyset$ iff $\mathrm{X}$ has the weak $B_{1}$-insertion property for $\left(u s B_{1}, l s B_{1}\right)$.

Proof. Let $\mathrm{g}$ and $\mathrm{f}$ be real-valued functions defined on the $X$, such that $f$ is $l s B_{1}, g$ is $u s B_{1}$, and $g \leq f$.If a binary relation $\rho$ is defined by $A \rho B$ in case $A^{\bar{\Lambda}} \subseteq B^{V}$, then by hypothesis $\rho$ is a strong binary relation in the power set of $X$. If $t_{1}$ and $t_{2}$ are any elements of $Q$ with $t_{1}<t_{2}$, then

$$
A\left(f, t_{1}\right) \subseteq\left\{x \in X: f(x) \leq t_{1}\right\} \subseteq
$$




$$
\left\{x \in X: g(x)<t_{2}\right\} \subseteq A\left(g, t_{2}\right) ;
$$

since $\left\{x \in X: f(x) \leq t_{1}\right\}$ is a $G_{\delta}$-set and since $\{x \in$ $\left.X: g(x)<t_{2}\right\}$ is a $F_{\sigma}-$ set, it follows that $A\left(f, t_{1}\right)^{\Lambda} \subseteq$ $A\left(g, t_{2}\right)^{V}$. Hence $t_{1}<t_{2}$ implies that $A\left(f, t_{1}\right) \rho A\left(g, t_{2}\right)$. The proof follows from Theorem 2. 1 .

On the other hand, let $G_{1}$ and $G_{2}$ are disjoint $G_{\delta}$-sets. Set $f=\chi_{G_{1}^{c}}$ and $g=\chi_{G_{2}}$, then $f$ is $l s B_{1}, g$ is $u s B_{1}$, and $g \leq f$. Thus there exists Baire-one function $h$ such that $g \leq h \leq f$. Set $F_{1}=\left\{x \in X: h(x)<\frac{1}{2}\right\}$ and $F_{2}=\left\{x \in X: h(x)>\frac{1}{2}\right\}$, then $F_{1}$ and $F_{2}$ are disjoint $F_{\sigma}$-sets such that $G_{1} \subseteq F_{1}$ and $G_{2} \subseteq F_{2}$.

Before stating the consequences of theorem 2.2, we state and prove the necessary lemmas.

Lemma 3.2. The following conditions on the space $X$ are equivalent:

(i) Every two disjoint $G_{\delta}$-sets of $X$ can be separated by $F_{\sigma}$-sets of $X$.

(ii) If $G$ is a $G_{\delta}$-set of $X$ which is contained in a $F_{\sigma}$-set $F$, then there exists a $F_{\sigma}$-set $H$ such that $G \subseteq$ $H \subseteq H^{\Lambda} \subseteq F$.

Proof. (i) $\Rightarrow$ (ii) Suppose that $G \subseteq F$, where $G$ and $F$ are $G_{\delta}$-set and $F_{\sigma}$-set of $X$, respectively. Hence, $F^{c}$ is a $G_{\delta}$-set and $G \cap F^{c}=\emptyset$.

By (i) there exists two disjoint $F_{\sigma}$-sets $F_{1}, F_{2}$ s.t. $G \subseteq F_{1}$ and $F^{c} \subseteq F_{2}$. But

$$
F^{c} \subseteq F_{2} \Rightarrow F_{2}^{c} \subseteq F
$$

and

$$
F_{1} \cap F_{2}=\emptyset \Rightarrow F_{1} \subseteq F_{2}^{c}
$$

hence

$$
G \subseteq F_{1} \subseteq F_{2}^{c} \subseteq F
$$

and since $F_{2}^{c}$ is a $G_{\delta}$-set containing $F_{1}$ we conclude that $F_{1}^{\Lambda} \subseteq F_{2}^{c}$, i.e.,

$$
G \subseteq F_{1} \subseteq F_{1}^{\Lambda} \subseteq F
$$

By setting $H=F_{1}$, condition (ii) holds.

(ii) $\Rightarrow$ (i) Suppose that $G_{1}, G_{2}$ are two disjoint $G_{\delta}-$ sets of $X$.

This implies that $G_{1} \subseteq G_{2}^{c}$ and $G_{2}^{c}$ is a $F_{\sigma}$-set. Hence by (ii) there exists a $F_{\sigma}-$ set $H$ s.t., $G_{1} \subseteq H \subseteq H^{\Lambda} \subseteq G_{2}^{c}$. But

$$
H \subseteq H^{\Lambda} \Rightarrow H \cap\left(H^{\Lambda}\right)^{c}=\emptyset
$$

and

$$
H^{\Lambda} \subseteq G_{2}^{c} \Rightarrow G_{2} \subseteq\left(H^{\Lambda}\right)^{c}
$$

Furthermore, $\left(H^{\Lambda}\right)^{c}$ is a $F_{\sigma}$-set of $X$. Hence $G_{1} \subseteq H, G_{2} \subseteq\left(H^{\Lambda}\right)^{c}$ and $H \cap\left(H^{\Lambda}\right)^{c}=\emptyset$. This means that condition (i) holds.

Lemma 3.3. Suppose that $X$ is the topological space s.t. we can separate every two disjoint $G_{\delta}$-sets by $F_{\sigma}$-sets. If $G_{1}$ and $G_{2}$ are two disjoint $G_{\delta}$-sets of $X$, then there exists a Baire-one function $h: X \rightarrow R$ s.t. $h\left(G_{1}\right)=\{0\}$ and $h\left(G_{2}\right)=\{1\}$.

Proof. Suppose $G_{1}$ and $G_{2}$ are two disjoint $G_{\delta}$-sets of $X$. Since $G_{1} \cap G_{2}=\emptyset$, hence $G_{1} \subseteq G_{2}^{c}$. In particular, since $G_{2}^{c}$ is a $F_{\sigma}$-set of $X$ containing $G_{1}$, by Lemma 3.2, there exists a $F_{\sigma}$-set $H_{1 / 2}$ s.t.,

$$
G_{1} \subseteq H_{1 / 2} \subseteq H_{1 / 2}^{\Lambda} \subseteq G_{2}^{c}
$$

Note that $H_{1 / 2}$ is a $F_{\sigma}$-set and contains $G_{1}$, and $G_{2}^{c}$ is a $F_{\sigma}$-set and contains $H_{1 / 2}^{\Lambda}$. Hence, by Lemma 3.2, there exists $F_{\sigma}$-sets $H_{1 / 4}$ and $H_{3 / 4}$ s.t.,

$$
G_{1} \subseteq H_{1 / 4} \subseteq H_{1 / 4}^{\Lambda} \subseteq H_{1 / 2} \subseteq H_{1 / 2}^{\Lambda} \subseteq H_{3 / 4} \subseteq H_{3 / 4}^{\Lambda} \subseteq G_{2}^{c}
$$

By continuing this method for every $t \in D$, where $D \subseteq[0,1]$ is the set of rational numbers that their denominators are exponents of 2 , we obtain $F_{\sigma}$-sets $H_{t}$ with the property that if $t_{1}, t_{2} \in D$ and $t_{1}<t_{2}$, then $H_{t_{1}} \subseteq H_{t_{2}}$. We define the real-valued function $h$ on $X$ by $h(x)=\inf \left\{t: x \in H_{t}\right\}$ for $x \notin G_{2}$ and $h(x)=1$ for $x \in G_{2}$.

Note that for every $x \in X, 0 \leq h(x) \leq 1$. Also, we note that for any $t \in D, G_{1} \subseteq H_{t}$; hence $h\left(G_{1}\right)=\{0\}$. Furthermore, by definition, $h\left(G_{2}\right)=\{1\}$. It remains only to prove that $h$ is a Baire-one function on $X$. For every $\alpha \in R$, we have if $\alpha \leq 0$ then $\{x \in X: h(x)<\alpha\}=\emptyset$ and if $0<\alpha$ then $\{x \in X: h(x)<\alpha\}=\cup\left\{H_{t}: t<\alpha\right\}$, hence, they are $F_{\sigma}$-sets of $X$. Similarly, if $\alpha<0$ then $\{x \in X: h(x)>\alpha\}=X$ and if $0 \leq \alpha$ then $\{x \in X: h(x)>\alpha\}=\cup\left\{\left(H_{t}^{\Lambda}\right)^{c}: t>\alpha\right\}$ hence, every of them is a $F_{\sigma}$-set. Consequently $h$ is a Baire-one function.

Lemma 3.4. Suppose that $X$ is the topological space such that every two disjoint $G_{\delta}$-sets can be separated by $F_{\sigma}$-sets. The following conditions are equivalent:

(i) Every countable convering of $F_{\sigma}$-sets of $X$ has a refinement consisting of $F_{\sigma}$-sets s.t., for every $x \in X$, there exists a $F_{\sigma}$-set containing $x$ such that it intersects only finitely many members of the refinement.

(ii) Corresponding to every decreasing sequence $\left\{G_{n}\right\}$ of $G_{\delta}$-sets with empty intersection there exists a decreasing sequence $\left\{F_{n}\right\}$ of $F_{\sigma}$-sets s.t., $\bigcap_{n=1}^{\infty} F_{n}=\emptyset$ and for every $n \in N, G_{n} \subseteq F_{n}$.

Proof. (i) $\Rightarrow$ (ii). suppose that $\left\{G_{n}\right\}$ be a decreasing sequence of $G_{\delta}$-sets with empty intersection. Then $\left\{G_{n}^{c}: n \in N\right\}$ is a countable covering of $F_{\sigma}$-sets. By hypothesis (i) and Lemma 3.2, this covering has a refinement $\left\{V_{n}: n \in N\right\}$ s.t. every $V_{n}$ is a $F_{\sigma}$-set and $V_{n}^{\Lambda} \subseteq G_{n}^{c}$. By setting $F_{n}=\left(V_{n}^{\Lambda}\right)^{c}$, we obtain a decreasing sequence of $F_{\sigma}$-sets with the required properties.

(ii) $\Rightarrow$ (i). Now if $\left\{H_{n}: n \in N\right\}$ is a countable covering of $F_{\sigma}$-sets, we set for $n \in N, G_{n}=\left(\bigcup_{i=1}^{n} H_{i}\right)^{c}$. Then $\left\{G_{n}\right\}$ is a decreasing sequence of $G_{\delta}$-sets with empty intersection. By (ii) there exists a decreasing sequence $\left\{F_{n}\right\}$ consisting of $F_{\sigma}$-sets s.t., $\bigcap_{n=1}^{\infty} F_{n}=\emptyset$ and for every $n \in N, G_{n} \subseteq F_{n}$. Now we define the subsets $W_{n}$ of $X$ in the following manner:

$W_{1}$ is a $F_{\sigma}$-set of $X$ s.t. $F_{1}^{c} \subseteq W_{1}$ and $W_{1}^{\Lambda} \cap G_{1}=\emptyset$.

$W_{2}$ is a $F_{\sigma}$-set of $X$ s.t. $W_{1}^{\bar{\Lambda}} \cup F_{2}^{c} \subseteq W_{2}$ and $W_{2}^{\Lambda} \cap$ $G_{2}=\emptyset$, and so on. (By Lemma 3.2, $W_{n}$ exists).

Then since $\left\{F_{n}^{c}: n \in N\right\}$ is a covering for $X$, hence $\left\{W_{n}: n \in N\right\}$ is a covering for $X$ consisting of $F_{\sigma}-$ sets.

Moreover, we have

(i) $W_{n}^{\Lambda} \subseteq W_{n+1}$

(ii) $F_{n}^{c} \subseteq W_{n}$ 
(iii) $W_{n} \subseteq \bigcup_{i=1}^{n} H_{i}$.

Now suppose that $S_{1}=W_{1}$ and for $n \geq 2$, we set $S_{n}=$ $W_{n+1} \backslash W_{n-1}^{\Lambda}$.

Then since $W_{n-1}^{\Lambda} \subseteq W_{n}$ and $S_{n} \supseteq W_{n+1} \backslash W_{n}$, it follows that $\left\{S_{n}: n \in N\right\}$ consists of $F_{\sigma}$-sets and covers $X$. Furthermore, $S_{i} \cap S_{j} \neq \emptyset$ iff $|i-j| \leq 1$. Finally, consider the following sets:

$$
\begin{aligned}
& S_{1} \cap H_{1}, \quad S_{1} \cap H_{2} \\
& S_{2} \cap H_{1}, \quad S_{2} \cap H_{2}, \quad S_{2} \cap H_{3} \\
& S_{3} \cap H_{1}, \quad S_{3} \cap H_{2}, \quad S_{3} \cap H_{3}, \quad S_{3} \cap H_{4}
\end{aligned}
$$

and continue ad infinitum. These sets are $F_{\sigma}$-sets, cover $X$ and refine $\left\{H_{n}: n \in N\right\}$. In addition, $S_{i} \cap H_{j}$ can intersect at most the sets in its row, immediately above, or immediately below row.

Hence if $x \in X$ and $x \in S_{n} \cap H_{m}$, then $S_{n} \cap H_{m}$ is a $F_{\sigma}$-set containing $x$ that intersects at most finitely many of sets $S_{i} \cap H_{j}$. Consequently, $\left\{S_{i} \cap H_{j}: i \in N, j=1, \ldots, i+1\right\}$ refines $\left\{H_{n}: n \in N\right\}$ s.t. its elements are $F_{\sigma}$-sets, and for every point in $X$ we can find a $F_{\sigma}$-set containing the point that intersects only finitely many elements of that refinement.

Remark 2. [2] , [3]. A space $X$ has the $c$-insertion property for $(u s c, l s c)$ iff $X$ is normal and countably paracompact.

Corollary 3.5. $X$ has the $B_{1}$-insertion property for $\left(u s B_{1}, l s B_{1}\right)$ iff every two disjoint $G_{\delta}-$ sets of $X$ can be separated by $F_{\sigma}$-sets, and in addition, every countable covering of $F_{\sigma}$-sets has a refinement that consists of $F_{\sigma}$-sets s.t., for every point of $X$ we can find a $F_{\sigma}$-set containing that point s.t., it intersects only a finite number of refining members.

Proof. Suppose that $G_{1}$ and $G_{2}$ are disjoint $G_{\delta}$-sets . Since $G_{1} \cap G_{2}=\emptyset$, it follows that $G_{2} \subseteq G_{1}^{c}$. We set $f(x)=2$ for $x \in G_{1}^{c}, f(x)=\frac{1}{2}$ for $x \notin G_{1}^{c}$, and $g=\chi_{G_{2}}$.

Since $G_{2}$ is a $G_{\delta}-$ set, and $G_{1}^{c}$ is a $F_{\sigma}$-set, therefore $g$ is $u s B_{1}, f$ is $l s B_{1}$ and furthermore $g<f$. Hence by hypothesis there exists a Baire-one function $h$ s.t., $g<$ $h<f$. Now by setting $F_{1}=\{x \in X: h(x)<1\}$ and $F_{2}=\{x \in X: h(x)>1\}$. We can say that $F_{1}$ and $F_{2}$ are disjoint $F_{\sigma}$-sets that contain $G_{1}$ and $G_{2}$, respectively. Now suppose that $\left\{G_{n}\right\}$ is a decreasing sequence of $G_{\delta}$-sets with empty intersection. Set $G_{0}=$ $X$ and define for every $x \in G_{n} \backslash G_{n+1}, f(x)=\frac{1}{n+1}$. Since $\bigcap_{n=0}^{\infty} G_{n}=\emptyset$ and for every $x \in X$, there exists $n \in N$, s.t., $x \in G_{n} \backslash G_{n+1}, f$ is well defined. Furthermore, for every $r \in R$, if $r \leq 0$ then $\{x \in X: f(x)>r\}=X$ is a $F_{\sigma}$-set and if $r>0$ then by Archimedean property of $R$, we can find $i \in N$ s.t. $\frac{1}{i+1} \leq r$. Now suppose that $k$ is the least natural number s.t. $\frac{1}{k+1} \leq r$. Hence $\frac{1}{k}>r$ and consequently, $\{x \in X: f(x)>r\}=X \backslash G_{k}$ is a $F_{\sigma}$-set. Therefore, $f$ is $l s B_{1}$. By setting $g=0$, we have $g$ is $u s B_{1}$ and $g<f$. Hence by hypothesis there exists a Baire-one function $h$ on $X$ s.t., $g<h<f$.

By setting $F_{n}=\left\{x \in X: h(x)<\frac{1}{n+1}\right\}$, we have $F_{n}$ is a $F_{\sigma}$-set. But for every $x \in G_{n}$, we have $f(x) \leq \frac{1}{n+1}$ and since $g<h<f$ therefore $0<h(x)<\frac{1}{n+1}$, i.e., $x \in F_{n}$ therefore $G_{n} \subseteq F_{n}$ and since $h>0$ it follows that $\bigcap_{n=1}^{\infty} F_{n}=\emptyset$. Hence by Lemma 3.4, the conditions holds.

On the other hand, since every two disjoint $G_{\delta}$-sets can be separated by $F_{\sigma}$-sets, therefore by Corollary 3.1, $X$ has the weak $B_{1}$-insertion property for $\left(u s B_{1}, l s B_{1}\right)$. Now suppose that $f$ and $g$ are real-valued functions on $X$ with $g<f$, s.t., $g$ is $u s B_{1}$ and $f$ is $l s B_{1}$. For every $n \in N$, set

$$
A\left(f-g, 3^{-n+1}\right)=\left\{x \in X:(f-g)(x) \leq 3^{-n+1}\right\} .
$$

Since $g$ is $u s B_{1}$, and $f$ is $l s B_{1}$, therefore $f-g$ is $l s B_{1}$. Hence $A\left(f-g, 3^{-n+1}\right)$ is a $G_{\delta}-$ set of $X$. Consequently, $\left\{A\left(f-g, 3^{-n+1}\right)\right\}$ is a decreasing sequence of $G_{\delta}$-sets and furthermore since $0<f-g$, it follows that $\bigcap_{n=1}^{\infty} A\left(f-g, 3^{-n+1}\right)=\emptyset$. Now by Lemma 3.4, there exists a decreasing sequence $\left\{D_{n}\right\}$ of $F_{\sigma}$-sets s.t. $A\left(f-g, 3^{-n+1}\right) \subseteq D_{n}$ and $\bigcap_{n=1}^{\infty} D_{n}=\emptyset$. But by Lemma $3.3, A\left(f-g, 3^{-n+1}\right)$ and $X \backslash D_{n}$ of $G_{\delta}$-sets can be completely separated by Baire-one functions. Hence by Theorem 2.2, there exists a Baire-one function $h$ defined on $X$ s.t., $g<h<f$, i.e., $X$ has the $B_{1}$-insertion property for $\left(u s B_{1}, l s B_{1}\right)$.

Remark 3. [6]. A space $X$ has the weak $c$-insertion property for $(l s c, u s c)$ iff $X$ is extremally disconnected.

Corollary 3.6. For every $F$ of $F_{\sigma}-$ set, $F^{\Lambda}$ is a $F_{\sigma}$-set iff $X$ has the weak $B_{1}$-insertion property for $\left(l s B_{1}, u s B_{1}\right)$.

Before giving the proof of this corollary, the necessary lemma is stated.

Lemma 3.7. The following conditions on the space $X$ are equivalent:

(i) For every $F$ of $F_{\sigma}$-set we have $F^{\Lambda}$ is a $F_{\sigma}$-set.

(ii) For each pair of disjoint $F_{\sigma}$-sets as $F_{1}$ and $F_{2}$ we have $F_{1}^{\Lambda} \cap F_{2}^{\Lambda}=\emptyset$.

The proof of lemma 3.7 is a direct consequence of the definition $\Lambda$-sets.

We now give the proof of corollary 3.6.

Proof. Let $\mathrm{g}$ and $\mathrm{f}$ be real-valued functions defined on the $X$, such that $f$ is $l s B_{1}, g$ is $u s B_{1}$, and $f \leq g$.If a binary relation $\rho$ is defined by $A \rho B$ in case $A^{\Lambda} \subseteq F \subseteq$ $F^{\Lambda} \subseteq B^{V}$ for some $F_{\sigma}-$ set $F$ in $X$, then by hypothesis and Lemma $3.7 \rho$ is a strong binary relation in the power set of $X$. If $t_{1}$ and $t_{2}$ are any elements of $Q$ with $t_{1}<t_{2}$, then

$$
\begin{gathered}
A\left(g, t_{1}\right)=\left\{x \in X: g(x)<t_{1}\right\} \subseteq\left\{x \in X: f(x) \leq t_{2}\right\} ; \\
=A\left(f, t_{2}\right) ;
\end{gathered}
$$

since $\left\{x \in X: g(x)<t_{1}\right\}$ is a $F_{\sigma}$-set and since $\{x \in$ $\left.X: f(x) \leq t_{2}\right\}$ is a $G_{\delta}$-set, by hypothesis it follows that $A\left(g, t_{1}\right) \rho A\left(f, t_{2}\right)$. The proof follows from Theorem 2.1.

On the other hand, Let $F_{1}$ and $F_{2}$ are disjoint $F_{\sigma}$-sets. Set $f=\chi_{F_{2}}$ and $g=\chi_{F_{1}^{c}}$, then $f$ is $l s B_{1}, g$ is $u s B_{1}$, and $f \leq g$.

Thus there exists Baire-one function $h$ such that $f \leq h \leq g$. Set $G_{1}=\left\{x \in X: h(x) \leq \frac{1}{3}\right\}$ and $G_{2}=\{x \in X: h(x) \geq 2 / 3\}$ then $G_{1}$ and $G_{2}$ are disjoint $G_{\delta}$-sets such that $F_{1} \subseteq G_{1}$ and $F_{2} \subseteq G_{2}$. Hence $F_{1}^{\Lambda} \cap F_{2}^{\Lambda}=\emptyset$.

Remark 4. [4]. A space $X$ has the $c$-insertion property for $(l s c, u s c)$ iff $X$ is extremally disconnected 
and if for any decreasing sequence $\left\{G_{n}\right\}$ of open subsets of $X$ with empty intersection there exists a decreasing sequence $\left\{F_{n}\right\}$ of closed subsets of $X$ with empty intersection such that $G_{n} \subseteq F_{n}$ for each $n$.

Corollary 3.8. For every $F$ of $F_{\sigma}-$ set, $F^{\Lambda}$ is a $F_{\sigma}$-set and in addition for every decreasing sequence $\left\{F_{n}\right\}$ of $F_{\sigma}$-sets with empty intersection, there exists a decreasing sequence $\left\{G_{n}\right\}$ of $G_{\delta}$-sets with empty intersection s.t. for every $n \in N, F_{n} \subseteq G_{n}$ iff $X$ has the $B_{1}$-insertion property for $\left(l s B_{1}, u s B_{1}\right)$.

Proof. Since for every $F$ of $F_{\sigma}$-set, $F^{\Lambda}$ is a $F_{\sigma}$-set, therefore by corollary 3.6, $X$ has the weak $B_{1}$-insertion property for $\left(l s B_{1}, u s B_{1}\right)$. Now suppose that $f$ and $g$ are real-valued functions defined on $X$ with $g<f, g$ is $l s B_{1}$, and $f$ is $u s B_{1}$. Set $A\left(f-g, 3^{-n+1}\right)=\{x \in X$ : $\left.(f-g)(x)<3^{-n+1}\right\}$. Then since $f-g$ is $u s B_{1}$, hence $\left\{A\left(f-g, 3^{-n+1}\right)\right\}$ is a decreasing sequence of $F_{\sigma}$-sets with empty intersection. By hypothesis, there exists a decreasing sequence $\left\{D_{n}\right\}$ of $G_{\delta}$-sets with empty intersection s.t., for every $n \in N, A\left(f-g, 3^{-n+1}\right) \subseteq D_{n}$. Hence $X \backslash D_{n}$ and $A\left(f-g, 3^{-n+1}\right)$ are two disjoint $F_{\sigma}$-sets and therefore by Lemma 3.7 , we have

$$
A\left(f-g, 3^{-n+1}\right)^{\Lambda} \cap\left(X \backslash D_{n}\right)^{\Lambda}=\emptyset
$$

and therefore by Lemma $3.3, X \backslash D_{n}$ and $A\left(f-g, 3^{-n+1}\right)$ are completely separable by Baire-one functions. Therefore by Theorem 2.2, there exists a Baire-one function $h$ on $X$ s.t., $g<h<f$, i.e., $X$ has the $B_{1}$-insertion property for $\left(l s B_{1}, u s B_{1}\right)$.

On the other hand, suppose that $F_{1}$ and $F_{2}$ be two disjoint $F_{\sigma}$-sets. Since $F_{1} \cap F_{2}=\emptyset$. We have $F_{2} \subseteq F_{1}^{c}$. We set $f(x)=2$ for $x \in F_{1}^{c}, f(x)=\frac{1}{2}$ for $x \notin F_{1}^{c}$ and $g=\chi_{F_{2}}$.

Then since $F_{2}$ is a $F_{\sigma}$-set and $F_{1}^{c}$ is a $G_{\delta}$-set, we conclude that $g$ is $l s B_{1}$ and $f$ is $u s B_{1}$ and furthermore $g<f$. By hypothesis, there exists a Baireone function $h$ on $X$ s.t., $g<h<f$. Now we set $G_{1}=\left\{x \in X: h(x) \leq \frac{3}{4}\right\}$ and $G_{2}=\{x \in X: h(x) \geq 1\}$. Then $G_{1}$ and $G_{2}$ are two disjoint $G_{\delta}$-sets contain $F_{1}$ and $F_{2}$, respectively. Hence $F_{1}^{\Lambda} \subseteq G_{1}$ and $F_{2}^{\Lambda} \subseteq G_{2}$ and consequently $F_{1}^{\Lambda} \cap F_{2}^{\Lambda}=\emptyset$. By Lemma 3.7 , for every $F$ of $F_{\sigma}$-set, the set $F^{\Lambda}$ is a $F_{\sigma}$-set.

Now suppose that $\left\{F_{n}\right\}$ is a decreasing sequence of $F_{\sigma}$-sets with empty intersection.

We set $F_{0}=X$ and $f(x)=\frac{1}{n+1}$ for $x \in F_{n} \backslash F_{n+1}$. Since $\bigcap_{n=0}^{\infty} F_{n}=\emptyset$ and for every $n \in N$ there exists $x \in F_{n} \backslash F_{n+1}, f$ is well-defined. Furthermore, for every $r \in R$, if $r \leq 0$ then $\{x \in X: f(x)<r\}=\emptyset$ is a $F_{\sigma}$-set and if $r>0$ then by Archimedean property of $R$, there exists $i \in N$ s.t. $\frac{1}{i+1} \leq r$. Suppose that $k$ is the least natural number with this property. Hence $\frac{1}{k}>r$. Now if $\frac{1}{k+1}<r$ then $\{x \in X: f(x)<r\}=F_{k}$ is a $F_{\sigma}$-set and if $\frac{1}{k+1}=r$ then $\{x \in X: f(x)<r\}=F_{k+1}$ is a $F_{\sigma}$-set. Hence $f$ is a $u s B_{1}$ on $X$. By setting $g=0$, we have conclude that $g$ is $l s B_{1}$ on $X$ and in addition $g<f$. By hypothesis there exists a Baire-one function $h$ on $X$ s.t., $g<h<f$.

Set $G_{n}=\left\{x \in X: h(x) \leq \frac{1}{n+1}\right\}$. This set is a $G_{\delta}$-set. But for every $x \in F_{n}$, we have $f(x) \leq \frac{1}{n+1}$ and since $g<h<f$ thus $h(x)<\frac{1}{n+1}$, this means that $x \in G_{n}$ and consequently $F_{n} \subseteq G_{n}$.

By definition of $G_{n},\left\{G_{n}\right\}$ is a decreasing sequence of $G_{\delta}$-sets and since $h>0, \bigcap_{n=1}^{\infty} G_{n}=\emptyset$. Thus the conditions holds.

\section{Acknowledgements}

This research was partially supported by Centre of Excellence for Mathematics(University of Isfahan).

\section{References}

[1] . Brooks, Indefinite cut sets for real functions, Amer. Math. Monthly, 78(1971), 1007-1010.

[2] . Katětov, On real-valued functions in topological spaces, Fund. Math., 38(1951), 85-91.

[3] . Katětov, Correction to, "On real-valued functions in topological spaces", Fund. Math., 40(1953), 203205.

[4] . Lane, Insertion of a continuous function, Pacific J. Math., 66(1976), 181-190.

[5] . Maki, Generalized $\Lambda$-sets and the associated closure operator, The special Issue in commemoration of Prof. Kazuada IKEDA's Retirement, (1986), 139146.

[6] .H. Stone, Boundedness properties in functionlattices, Canad. J. Math., 1(1949), 176-189. 